\title{
Multifunctional Composites with Intrinsic Pressure Actuation and Prestress for Morphing Structures
}

\author{
V.S.C. Chillara, L.M. Headings, M.J. Dapino* \\ Department of Mechanical and Aerospace Engineering, The Ohio State University, \\ Columbus, $\mathrm{OH}$ 43210, USA
}

\begin{abstract}
Pressure-actuated elements can be embedded in morphing panels to achieve continuous control of shape and stiffness. This paper presents a multifunctional laminated composite that exhibits a curved geometry due to intrinsic mechanical prestress and a change in curvature when fluid (liquid or gas) contained in one of its laminae is pressurized. The composite is composed of a mechanically-prestressed layer, a fluidic layer, and a constraining layer. The composite can be driven to any desired shape up to a flat limiting shape through modulation of pressure in its fluidic layer. An analytical model is developed to characterize the quasi-static response of such a composite to the applied fluid pressure for various laminate stacking sequences. A parametric study is also conducted to study the effects of the dimensions of the fluid channel and its spatial location. Composite beams are fabricated in the laminate configuration that requires the least actuation effort for a given change in curvature. Pneumatic pressure is applied to the composite in an open-loop setup and its response is measured using a motion capture system. The simulated response of the composite is in agreement with the measured response.
\end{abstract}

Key words:

morphing, prestressed, fiber-reinforced elastomer, active composite, pressure-actuated

\footnotetext{
* Corresponding author

Email addresses: chillara.1@osu.edu (V.S.C. Chillara), headings.4@osu.edu (L.M. Headings), dapino.1@osu.edu (M.J. Dapino)
} 


\section{Introduction}

Morphing structures have gained importance in the aerospace and automotive industries due to their ability to serve multiple functions as adaptive control surfaces. In an aircraft, wings with variable-geometry optimize aerodynamic drag at various operating conditions [1], leading to improved fuel economy and performance. Morphing concepts for aircraft wings have been studied extensively $[2,3]$. In automobiles, potentially conflicting requirements like aesthetics and aerodynamic efficiency can be met by incorporating morphing panels into the vehicle body [4]. A design requirement encountered quite often in the development of morphing panels is the ability to achieve a large change in curvature. Curvature change is typically achieved by actuating a panel whose stiffness is tailored for the desired shape transition.

Curvature in a fiber-reinforced polymeric (FRP) composite can be created by imparting residual stress in its matrix through high temperature curing $[5,6]$. In symmetric FRP laminates, creation of curvature requires a mechanically applied fiber-prestress in addition to thermally-induced matrix prestress $[7,8]$. Initially-curved composites with a mechanically-prestressed matrix can serve as morphing elements when installed in a structure [9]. The limitation in the existing designs is that the entire matrix must be prestressed to create curvature. Application of matrix prestress in specific laminae allows combination with a wide variety of other laminae to create multifunctional composites. Chillara et al. [10] demonstrated a curved laminated composite with an intrinsic prestress in the matrix of a single lamina. In this design, the residual stress can only be redistributed within the composite and not relieved.

Embedded actuation is preferred for morphing panels due to the possibilities for reducing weight, size, and complexity. In most cases, the actuation material is either inserted into channels created in a passive composite [11] or is an active layer(s) in a laminated composite [12]. Passive composites can also be actuated through thermal loading [13, 14]. However, this method is mostly restricted to thermally-cured FRP laminates. Ideally, an actuation material embedded in a morphing composite should have a high power output per unit volume and operate the composite in a frequency range consistent with the structural dynamics. Piezoelectric materials can 
generate an adequate amount of force [15] but require stroke amplification to achieve large deflection while maintaining system rigidity and frequency response. Shape memory alloys can provide sufficient force and stroke although their application is often limited by their low operating frequency limit of about a few $\mathrm{Hz}$. Continuous shape control is a challenge in the case of shape memory materials. In some cases, hydraulic and pneumatic actuators can produce large force and stroke in the frequency range of the structure, but with a weight penalty. Lightweight and compact solutions for harnessing fluid power are offered by smart material-based miniature electrohydraulic actuators [16] that amplify the small stroke of materials with high frequency bandwidth such as piezoelectrics and magnetostrictives through fluid flow rectification.

Fluid-based actuation systems are of interest in fields like soft robotics, bio-inspired structures, and morphing structures. Linear actuators such as pressurized artificial muscles are often used to create robotic mechanisms that can bend or fold [17]. Flexible pneumatic bending actuators have been proposed by Deimel and Brock [18] for a soft-robotic hand that can grip objects. Marchese et al. [19] developed a compliant structure with embedded fluid channels that is capable of replicating fishlike motion. Philen et al. [20, 21] and Feng et al. [22] developed variable-stiffness skins with embedded fluidic muscles that can be used for morphing aircraft wings. The basic design principle in these fluidic actuators is that linear actuation is achieved by restricting radial expansion through fiber-reinforcement while bending is achieved by bonding a constraining layer to a linear actuator.

This paper presents a fluidic prestressed composite (FPC) in which fluid power is used to morph its shape from a curved to a flat geometry (Figure 1). Intermediate curvatures are obtained through the modulation of pressure of the contained fluid. A prestressed elastomeric layer, a fluidic layer, and a constraining layer constitute this composite. While the equilibrium shape of the proposed fluidic composite is created through application of mechanical prestress to the elastomeric layer (Figure 1(a)), morphing action is accomplished through pressurization of the fluidic layer of the composite (Figure $1(\mathrm{~b}))$.

An elastomeric matrix composite (EMC) exhibits anisotropic stiffness through fiber-reinforcement in an elastomeric matrix [23]. An EMC with unidirectional fibers in the transverse direction $\left(90^{\circ}\right)$ has been proposed as a flexible-skin panel for the span morphing 


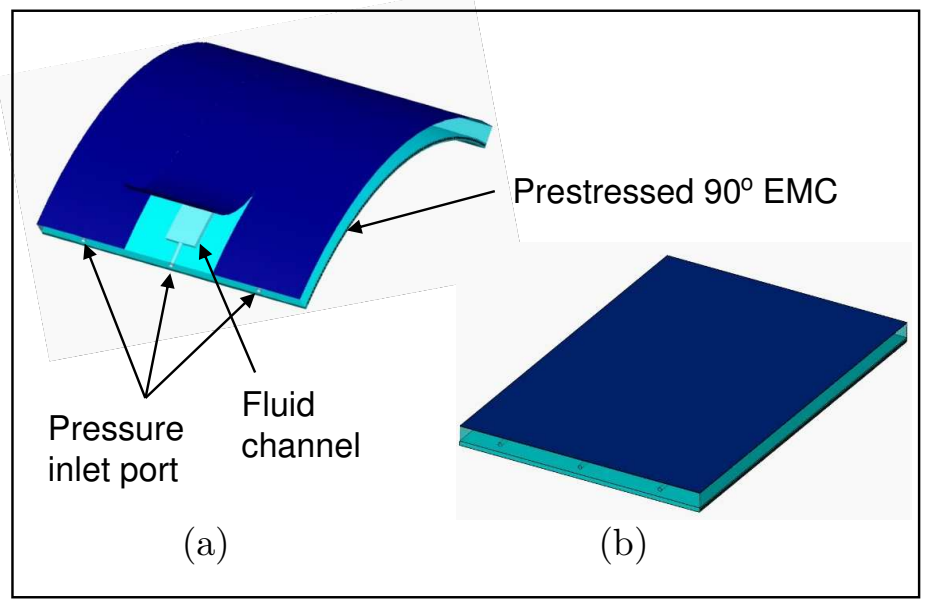

Figure 1: (a) Geometry of a fluidic prestressed composite in the unactuated state, (b) limiting actuated shape of the composite when the fluid channels are pressurized. EMC stands for elastomeric matrix composite.

of an aircraft wing $[23,24]$. The property of near-zero in-plane Poisson's ratio of a $90^{\circ} \mathrm{EMC}$ is used to create a single cylindrical curvature in the FPC. Daynes et al. [25] presented reinforced elastomers in a prestressed condition in a morphing wing structure but not in a laminated-composite setup.

An FPC (Figure 1) is capable of controlled shape transition from a cylindrical shell to a flat plate through simultaneous actuation of parallel fluid channels that are embedded along the curve. The actuation mechanism is shown in Figure 2(c). The fluid channels are molded into a reinforced flexible lamina instead of being embedded as individually-reinforced fluidic muscles in a flexible medium as in $[21,26]$, resulting in a simpler fabrication process. Further, molded fluid channels can have an arbitrary cross section, leading to lower composite thickness for a given actuation effort. Complex curvatures in an FPC are possible through a vascular network of fluid channels. Through the design of individual channel dimensions, the maximum force exerted along the fluid path and hence the localized curvature can be regulated. Multiple pressure sources enable sequential actuation of various regions of the composite.

The laminae of the composite and their stacking sequences are described 


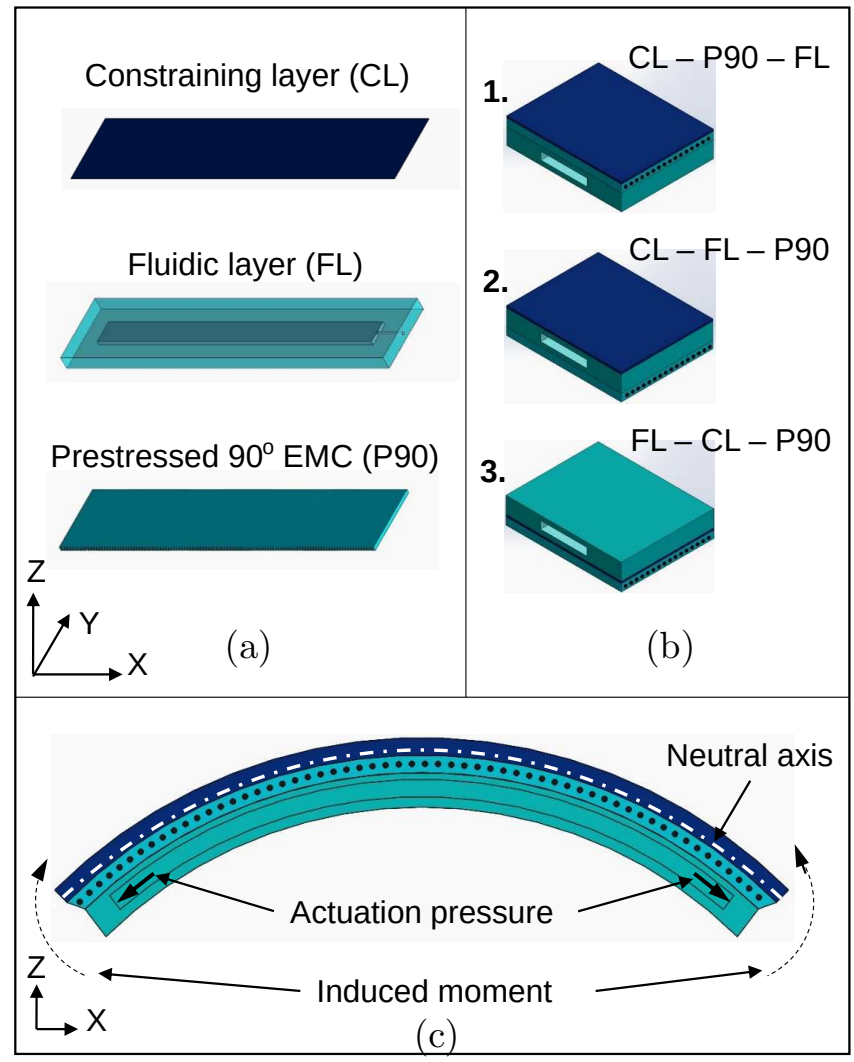

Figure 2: (a) Participating laminae, (b) possible laminate configurations of a fluidic prestressed composite, (c) for configuration 1, pressurization of the fluidic layer induces moments as shown.

in section 2. A nonlinear analytical laminated-plate model is developed to characterize the response of a generic FPC (section 3). The large-deflection problem is defined using a Lagrangian strain formulation based on classical laminated plate theory. Material and geometric nonlinearity of a prestressed EMC is included in this model. Quasi-static equilibrium curvature is calculated as a function of the applied fluid pressure by minimizing the total energy of the composite. The laminate configuration of a morphing panel that requires the least actuation effort is determined through a model-based analysis. A design methodology for the proposed composite is presented through a parametric study of the effect of fluid channel sizing on composite response (section 4). A technique for fabricating a fluidic prestressed com- 
posite is proposed and demonstrated (section 5). The fabrication method for the fluidic layer is inspired by fluidic elements designed for applications in soft robotics. The fabricated samples are pneumatically actuated and their quasi-static response is recorded using a motion capture system for model validation (section 6).

\section{Fluidic Prestressed Composite}

The laminae of a fluidic prestressed composite viz., prestressed elastomeric layer, fluidic layer, and constraining layer, are described in this section. Also, the possible laminate configurations, each resulting in a unique response of the composite, are introduced.

\subsection{Description of laminae}

An EMC is a fiber-reinforced elastomer whose material properties are influenced by the orientation and volume fraction of the embedded fibers. A $90^{\circ} \mathrm{EMC}$ has fibers oriented along the $Y$ axis $\left(90^{\circ}\right)$, making it stiff in this direction and stretchable in the orthogonal direction (Figure 2(a)). Large strain (up to 100\%) can be applied to a $90^{\circ}$ EMC in the $X$ direction while maintaining near-zero in-plane Poisson's ratio, provided the modulus of the fiber is much higher than that of the elastomer. In an FPC, a curved equilibrium shape is created by including a prestressed $90^{\circ} \mathrm{EMC}$ as one of the laminae. Prestress in this layer is created by applying the corresponding mechanical strain prior to bonding the layer.

A fluidic layer consists of fluid channels molded into a continuous flexible medium (Figure 2(a)). Stiffness of the fluidic layer must be tailored such that the fluid channel expands only along its length when the composite is pressurized. For a channel with a rectangular cross-section, strain in the $Y$ direction is negligible due to the constraint offered by the adjacent laminae, whereas strain in the $Z$ direction is finite and is mitigated through reinforcement of the unbonded face of the fluidic layer with unidirectional fibers in the $Y$ direction.

A constraining layer is typically very thin with its $X Y$ plane modulus being much higher than that of the other layers (Figure 2(a)). Its function is to constrain the length of the composite, thereby translating the effect of internal forces acting along its length into curvature about the $Y$ axis (Figure 2(c)). For morphing panels, this layer can be designed to add flexural stiffness to the composite. A constraining layer can also be built into the 
fluidic layer through suitable fiber-reinforcement to create bending actuators for soft robotics and biomimetics applications [27].

\subsection{Laminate configurations}

The laminae of a fluidic prestressed composite can be arranged in three unique configurations (Figure 2(b)). In configuration 1, a prestressed $90^{\circ}$ EMC is sandwiched between a constraining layer and a fluidic layer. The equilibrium shape of the composite in the unactuated state is such that the fluidic layer is in compression. Sandwiching a fluidic layer between a constraining layer and a prestressed $90^{\circ} \mathrm{EMC}$ as in configuration 2 also results in a curved composite where the fluidic layer is in a compressed state. In both of these arrangements, the effect of actuation is to elongate the fluidic layer, thereby flattening the composite. The actuation effort for a given change in curvature of the composite is different between these two configurations (discussed in section 4). In configuration 3, a constraining layer is located between a fluidic layer and a prestressed $90^{\circ} \mathrm{EMC}$, with the fluidic layer on the convex side of the composite at equilibrium. Pressurization of the fluid channel results in further bending of the composite. This configuration can also be realized without an EMC to generate curvature in an initially flat composite. To calculate the quasi-static curvature of an FPC, an analytical model is presented in the following section. While the proposed model is applicable to all three laminate configurations, configuration 1 is chosen for the purpose of presentation.

\section{Analytical Model}

Morphing of a fluidic prestressed composite is achieved with minimal actuation effort when the fluid channels are aligned with the direction of EMC prestrain. The simplest structure that reflects this condition is a beam in which prestrain in the $90^{\circ} \mathrm{EMC}$ is applied in the $X$ direction and the fluid channel is oriented along the $X$ axis. (Figure 3). An FPC beam is expected to have a single equilibrium curvature in the unactuated state about the $Y$ axis. Curvature about $X$ is negligible since the EMC has no prestress in the $Y$ direction (zero Poisson's ratio). Pressurization of the fluid channel does not affect the direction of composite curvature. The mechanics of an FPC beam are modeled analytically to study the influence 
of prestress in the $90^{\circ} \mathrm{EMC}$ and pressure in the fluid channel on its curvature. A nonlinear Lagrangian strain formulation is used in conjunction with classical laminate plate theory to quantify the strain energy of the composite. The fluidic and constraining layers are linearly elastic due to the relatively low in-plane strain associated with curvature in the composite. However, the $90^{\circ} \mathrm{EMC}$ has a nonlinear elastic behavior due to the large in-plane strain applied to it to create a prestressed condition. Actuation of the composite is modeled as the work done on the composite by the working fluid. A Rayleigh-Ritz method is employed to minimize the net energy of the composite and hence calculate the strain and curvature at quasi-static equilibrium.

\subsection{Strain energy of the composite}

Strain of the composite's mid-plane is described using unknown polynomial functions whose degree depends on the equilibrium shape. For example, in composites with thermally induced bistability where curvatures about two axes and out-of-plane twist are possible, third degree complete polynomials in $x$ and $y$ are used to describe axial strains (Hyer [28], Dano and Hyer [29]). Since the FPC beam (Figure 3 ) is expected to have a single curvature, simpler strain functions can be used. Longitudinal strain of the beam is described by a second-degree polynomial in $x$ whereas lateral strain is described by a constant. A second degree polynomial in $x$ is used to describe the out-of-plane displacement. Since the $90^{\circ} \mathrm{EMC}$ layer has a near-zero in-plane Poisson's ratio, curvature about the $X$ axis is negligible. Further, it is assumed that the Poisson's ratio difference between layers has no effect on the curvature about the $X$ axis. The stable shape is expected to be curved about the $Y$ axis such that the fluidic layer is under axial compression ( $X$-direction). Per the assumptions of classical laminate plate theory, transverse shear $(X Z, Y Z)$ and transverse normal $(Z Z)$ stresses are neglected. Based on von Karman's hypothesis [30], strains for composite 


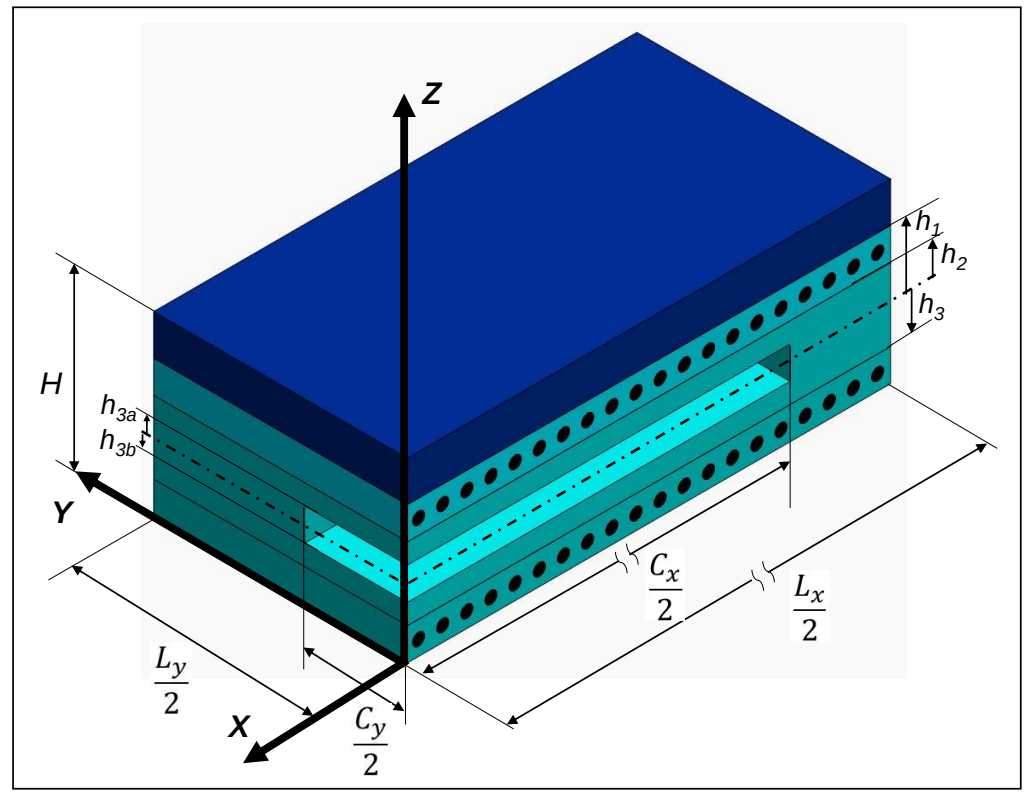

Figure 3: Geometry for a beam model of a fluidic prestressed composite illustrated in configuration 1. The bottom face of the fluidic layer is reinforced with unidirectional fibers oriented along the $Y$ direction.

materials with geometric nonlinearities, as applicable to this problem, are:

$$
\begin{aligned}
\epsilon_{x} & =\frac{\partial u}{\partial x}+\frac{1}{2}\left(\frac{\partial w}{\partial x}\right)^{2}, \\
\epsilon_{y} & =\frac{\partial v}{\partial y} \\
\gamma_{x y} & =\frac{\partial u}{\partial y}+\frac{\partial v}{\partial x}+\frac{\partial w}{\partial x} \frac{\partial w}{\partial y} .
\end{aligned}
$$

Displacements $u, v$, and $w$ of any point in the composite are written in terms of mid-plane displacements $u_{0}, v_{0}$, and $w_{0}$ in the $X, Y$, and $Z$ directions, respectively, as:

$$
\begin{aligned}
u(x, y, z) & =u_{0}(x)-z \frac{\partial w_{0}}{\partial x} \\
v(x, y, z) & =v_{0}(y) \\
w(x, y, z) & =w_{0}(x) .
\end{aligned}
$$


Substitution of (4) - (6) into (1) - (3) yields the strain of an arbitrary plane $z$ of the composite:

$$
\begin{aligned}
\epsilon_{x} & =\frac{\partial u_{0}}{\partial x}+\frac{1}{2}\left(\frac{\partial w_{0}}{\partial x}\right)^{2}-z\left(\frac{\partial^{2} w_{0}}{\partial x^{2}}\right), \\
\epsilon_{y} & =\frac{\partial v_{0}}{\partial y} \\
\gamma_{x y} & =\frac{\partial u_{0}}{\partial y}+\frac{\partial v_{0}}{\partial x}+\frac{\partial w}{\partial x} \frac{\partial w}{\partial y}-z\left(\frac{\partial^{2} w_{0}}{\partial y \partial x}\right) .
\end{aligned}
$$

The expression for strain of an arbitrary plane $z$ in the composite beam has the structure:

$$
\epsilon_{x}=\epsilon_{x}^{0}+z \kappa_{x}^{0}, \quad \epsilon_{y}=\epsilon_{y}^{0}, \quad \gamma_{x y}=\gamma_{x y}^{0}+z \kappa_{x y}^{0},
$$

where $\epsilon_{x}^{0}$ and $\epsilon_{y}^{0}$ are the in-plane axial strains, $\gamma_{x y}^{0}$ is the in-plane shear strain, and $\kappa_{x}^{0}$ and $\kappa_{x y}^{0}$ are the curvature and twist, respectively, of the mid-plane. From (7) and (10), it can be seen that $\kappa_{x}^{0}$ is the second derivative of the displacement function $w$ with respect to $x$. Assuming $\kappa_{x}^{0}$ to be constant throughout the mid-plane, a second degree polynomial in $x$ is sufficient to approximate the mid-plane displacement $w_{0}$ in the $Z$ direction:

$$
w_{0}(x)=\frac{1}{2} a x^{2},
$$

where $-a$ represents the curvature $\left(\kappa_{x}^{0}\right)$ about the $Y$ axis $[28,29]$. Strains in the $X$ and $Y$ directions are described using second and zero degree polynomials, respectively, as:

$$
\epsilon_{x}^{0}=c_{0}+c_{1} x+c_{2} x^{2} \quad \text { and } \quad \epsilon_{y}^{0}=d_{0}
$$

By inspection of (9), $\gamma_{x y}$ is zero because the polynomial functions that describe $u, v$, and $w$ are independent of $y, x$, and $y$ respectively.

The material properties of fluidic and constraining layers are considered to be linear for small in-plane strains of the composite. However, the mechanical behavior of a $90^{\circ} \mathrm{EMC}$ is nonlinear since a large prestrain is applied to it during fabrication. This includes a 1-D geometric nonlinearity due to large axial strain and material nonlinearity due to viscoelastic behavior of 
the EMC. The focus of our model is to incorporate the nonlinear mechanical behavior of an elastomer-like layer into the mechanics of a laminated composite that can be modeled using conventional laminate theories.

Peel and Jensen [31] developed a nonlinear model to describe the mechanics of fiber-reinforced elastomers. A similar method is employed to model the uniaxial response of a $90^{\circ} \mathrm{EMC}$. The expression for strain of a single $90^{\circ}$ EMC subjected to a large strain along $X$ is written as:

$$
e_{x}^{(90)}=\frac{\partial u}{\partial x}+\frac{1}{2}\left(\frac{\partial u}{\partial x}\right)^{2}
$$

where $e_{x}^{(90)}$ is the measured nonlinear strain and $u$ is the constant axial displacement of the EMC under a tensile test. Since the Lagrangian strain formulation presented in (7) has a linear axial strain term, the nonlinear strain of the $90^{\circ}$ EMC must be expressed in terms of linear strain so that it can be modeled as a layer in the composite. The expression for the corresponding linear axial strain is:

$$
\varepsilon_{x}^{(90)}=\frac{\partial u}{\partial x}
$$

The nonlinear strain $e_{x}^{(90)}$ is expressed as a quadratic function of $\varepsilon_{x}^{(90)}$ by substituting (14) in (13). Solving for $\varepsilon_{x}^{(90)}$ and discarding the negative root (non-physical solution), one obtains $\varepsilon_{x}^{(90)}$ as:

$$
\varepsilon_{x}^{(90)}=-1+\sqrt{1+2 e_{x}^{(90)}} .
$$

Based on linear strain, stress is calculated incrementally as:

$$
\sigma_{x}^{(90)}{ }_{i}=\sigma_{x}^{(90)}{ }_{i-1}+E_{i}\left(\varepsilon_{x}^{(90)}{ }_{i}-\varepsilon_{x}^{(90)}{ }_{i-1}\right),
$$

where $E_{i}$ is the point-wise modulus of a $90^{\circ}$ EMC (local slope of its stressstrain curve). Peel and Jensen [31] obtained the stress function by fitting experimental data to traditional hyperelastic models. For computational efficiency, the stress function is described by a polynomial. The design details of a tested $90^{\circ} \mathrm{EMC}$ are presented in section 5. Since the prestressed EMC is subject to loading and unloading during the operation of an FPC, the average stress from the hysteretic stress-strain 
curve is considered as the nonlinear stress function of the $90^{\circ} \mathrm{EMC}$ (Figure $4)$. By the method of least squares, the reduced stress function is described using a quartic polynomial as:

$$
\sigma_{x}^{(90)}=-0.698 \varepsilon_{x}^{(90)^{4}}+2.29 \varepsilon_{x}^{(90)^{3}}-2.306 \varepsilon_{x}^{(90)^{2}}+1.598 \varepsilon_{x}^{(90)} \quad[\mathrm{MPa}] .
$$

For a $90^{\circ} \mathrm{EMC}$, a polynomial stress function is preferred to traditional rubber models like the Ogden model [32] for the sake of computational efficiency. The stress function of an EMC depends on design parameters such as fiber volume fraction and orientation [33].

The total potential energy $(U)$ of the system can be expressed as the sum of the strain energy of the initially-stress-free host structure comprising of the fluidic $\left(U_{(F L)}\right)$ and constraining layers $\left(U_{(C L)}\right)$, and the residual strain energy $\left(U_{(P 90)}\right)$ of the $90^{\circ}$ EMC as:

$$
U=U_{(C L)}+U_{(F L)}+U_{(P 90)} .
$$

In practice, the bottom face of the fluidic layer in cases 1 and 3 (Figure 2(b)) is reinforced with fibers in the $90^{\circ}$ orientation to mitigate expansion in the thickness direction. In case 2, the face of the fluidic layer that is bonded to the prestressed EMC is reinforced with $90^{\circ}$ fibers. This step is equivalent to the addition of a stress-free $90^{\circ}$ EMC to the fluidic layer. The contribution of strain energy of the laminate adhesive to the total strain energy is assumed to be negligible. The total strain energy of the linear elastic layers can be written as:

$$
U_{(C L)}+U_{(F L)}=\int_{x_{l}}^{x_{u}} \int_{y_{l}}^{y_{u}} \int_{z_{l}}^{z_{u}}\left(\frac{1}{2} Q_{11} \epsilon_{x}^{2}+Q_{12} \epsilon_{x} \epsilon_{y}+\frac{1}{2} Q_{22} \epsilon_{y}^{2}\right) \mathrm{d} z \mathrm{~d} y \mathrm{~d} x,
$$

where $\left\{Q_{11}, Q_{12}\right.$, and $\left.Q_{22}\right\}$ are the plane stress-reduced stiffness parameters [30] defined as:

$$
Q_{11}=\frac{E_{1}}{1-\nu_{12} \nu_{21}}, \quad Q_{12}=\frac{\nu_{12} E_{2}}{1-\nu_{12} \nu_{21}}, \quad Q_{22}=\frac{E_{2}}{1-\nu_{12} \nu_{21}},
$$

and $E$ and $\nu$ are the elastic modulus and Poisson's ratio, respectively. The limits of integration that must be applied for each layer in the host structure are listed in Table 1. 
Table 1: Limits of integration for the computation of strain energy of the linear elastic layers of the composite.

\begin{tabular}{cccccc}
\hline Coordinate & Constraining layer & Prestressed $90^{\circ}$ EMC & Fluidic layer & Fluid channel & $\begin{array}{c}90^{\circ} \text { fiber-reinforcement } \\
\text { in fluidic layer }\end{array}$ \\
\hline$x$ & $\left(-L_{x} / 2, L_{x} / 2\right)$ & $\left(-L_{x} / 2, L_{x} / 2\right)$ & $\left(-L_{x} / 2, L_{x} / 2\right)$ & $\left(-C_{x} / 2, C_{x} / 2\right)$ & $\left(-L_{x} / 2, L_{x} / 2\right)$ \\
$y$ & $\left(-L_{y} / 2, L_{y} / 2\right)$ & $\left(-L_{y} / 2, L_{y} / 2\right)$ & $\left(-L_{y} / 2, L_{y} / 2\right)$ & $\left(-C_{y} / 2, C_{y} / 2\right)$ & $\left(-L_{y} / 2, L_{y} / 2\right)$ \\
$\left(h_{1}, H / 2\right)$ & $\left(h_{2}, h_{1}\right)$ & $\left(-h_{3}, h_{2}\right)$ & $\left(-h_{3 b}, h_{3 a}\right)$ & $\left(-H / 2,-h_{3}\right)$ \\
\hline $\begin{array}{c}\text { Strain } \\
\text { energy }\end{array}$ & $U_{(C L)}$ & $U_{(P 90)}$ & $\begin{array}{c}U_{(\text {layer })} \\
U_{(F L)}=U_{(\text {layer })}-U_{(\text {channel) }}+U_{(\text {reinforcement })}\end{array}$ \\
\hline
\end{tabular}

The strain energy of a prestressed $90^{\circ}$ EMC is expressed as:

$$
U_{(P 90)}=\int_{x_{l}}^{x_{u}} \int_{y_{l}}^{y_{u}} \int_{z_{l}}^{z_{u}}\left(U_{(P 90)}^{x}+\frac{1}{2} Q_{22} \epsilon_{y}^{2}\right) \mathrm{d} z \mathrm{~d} y \mathrm{~d} x
$$

where $U_{(P 90)}^{x}$ is the strain energy in the $X$ direction, calculated as the area under a nonlinear averaged stress-strain curve obtained from a uniaxial tensile test (Figure 4). The resulting strain energy in the prestressed $90^{\circ} \mathrm{EMC}$ as a function of strain of the composite and layer prestress $\left(\epsilon_{0}\right)$, is of the form:

$$
U_{(P 90)}=f\left(\epsilon_{0}-\epsilon_{x}, \epsilon_{y}\right) .
$$

\subsection{Work done by applied fluid pressure}

Pressurization of a working fluid in the fluidic layer results in actuation of the composite. The work done by a fluid on the composite can be expressed as a product of the operating pressure and the change in volume of the fluid channel due to the applied pressure. While the initial volume $\left(V_{i}\right)$ of the fluid channel corresponds to the volume at static equilibrium, the final volume $\left(V_{f}\right)$ is a function of the unknown strain functions in $x$ and $y$. Since the constant initial volume vanishes in the energy minimization step, it is not a critical element in the computation of composite geometry. However, it is required in the calculation of actuation power. In this model, it is assumed that volume change occurs in-plane; thickness of the composite remains constant. Assuming that the working fluid is an ideal gas operating in adiabatic conditions, work done on the composite [34] by pneumatic actuation can be expressed as:

$$
W=\frac{P_{i} V_{i}-P_{f} V_{f}}{\gamma-1}
$$




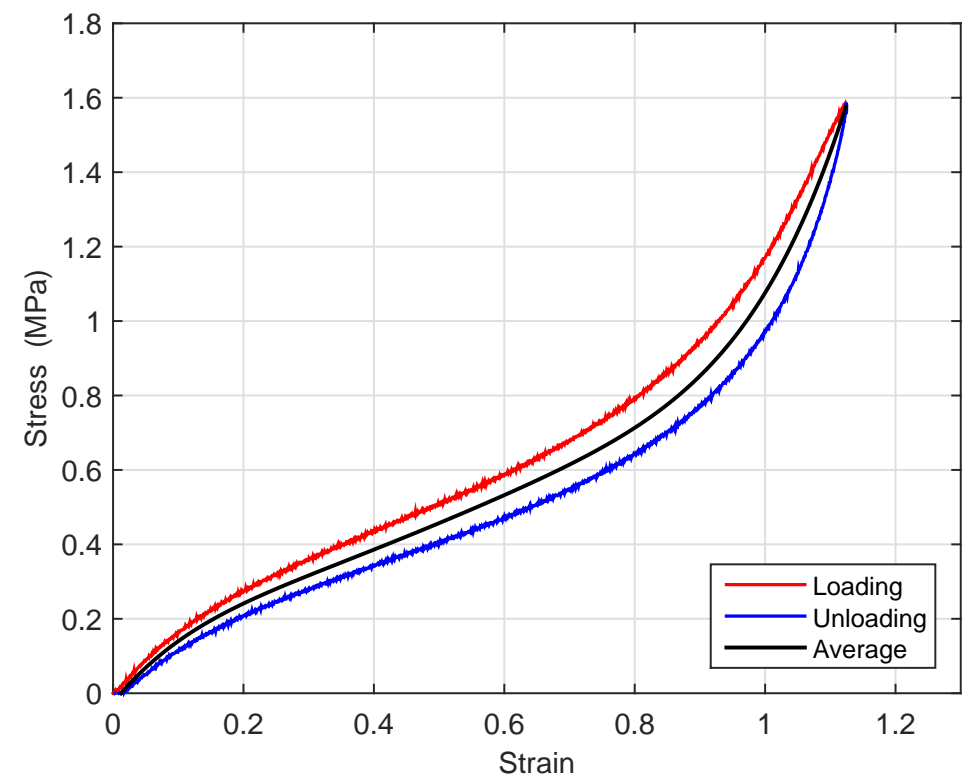

Figure 4: Stress-strain curve for a $90^{\circ}$ EMC constructed as described in section 5.1, obtained from a uniaxial tensile test.

where $\gamma=1.4$ is the adiabatic coefficient of air. The volume $\left(V_{f}\right)$ of the fluid channel in the actuated state can be expressed in terms of the unknown strain polynomials as:

$$
\begin{aligned}
V_{f} & =\int_{V}\left(1+\epsilon_{x}\right)\left(1+\epsilon_{y}\right) \mathrm{d} V, \\
& =\int_{V}\left(1+c_{0}+c_{1} x+c_{2} x^{2}+a z\right)\left(1+d_{0}\right) \mathrm{d} V .
\end{aligned}
$$

\subsection{Computation of composite shape}

The equilibrium shape of the morphing composite is determined using the principle of virtual work. Exact differentials of the strain energy and work terms formed with respect to the unknown constants of strain polynomials are written as:

$$
\sum_{k} \frac{\partial(U-W)}{\partial k} \delta k=0, \quad k=\left\{a, c_{0}, c_{1}, c_{2}, d_{0}\right\} .
$$


The partial derivatives of the strain energy and work terms are computed symbolically to obtain five nonlinear algebraic equations. These equations are solved numerically using the Newton-Raphson technique (or similar). The model presented thus far provides a means to design the curvature of an FPC in its unactuated state and the required actuation pressure range to obtain a flat limiting shape. The following section is a study of the response of an FPC in each of its laminate configurations.

\section{Model-Based Study of Composite Response}

The effect of laminate stacking sequence on the quasi-static response of an FPC is investigated using the model presented in section 3. The three layer arrangements in Figure 2(b) are considered with the dimensions shown in Figure 5 and the material properties listed in Table 2. The configuration that results in the least actuation effort for a given change in curvature is identified. The influence of design parameters such as the size and location of the fluid channel on composite response is examined through a parametric study.

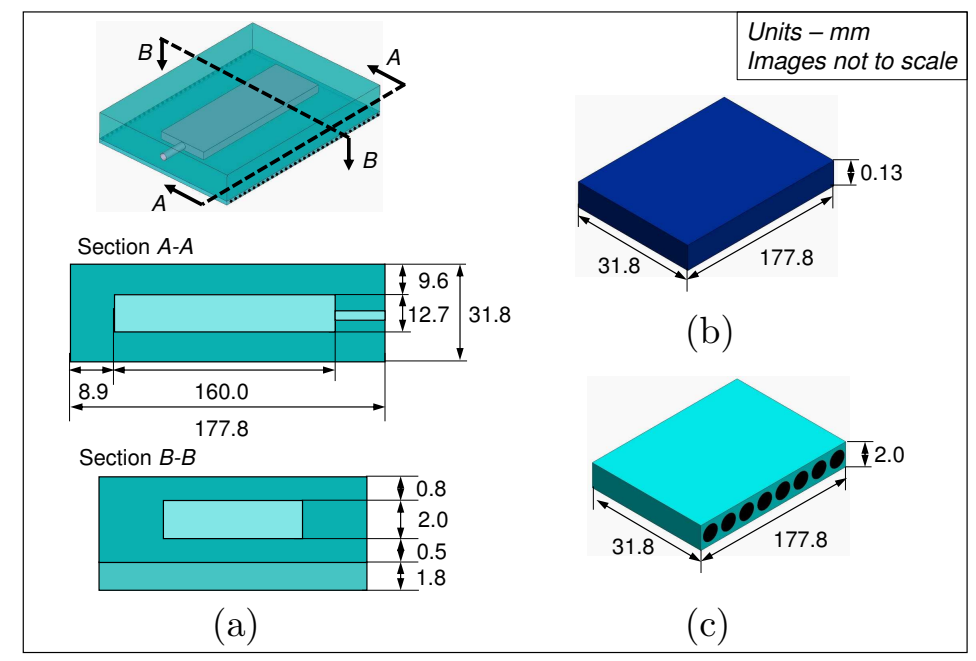

Figure 5: Dimensions of the laminae of a fluidic prestressed composite: (a) fluidic layer, (b) constraining layer, and (c) prestressed $90^{\circ}$ EMC. 
Table 2: Measured material properties of the laminae of a fluidic prestressed composite.

\begin{tabular}{lrrrr}
\hline \multicolumn{1}{c}{ Lamina } & $E_{x}(\mathrm{MPa})$ & $E_{y}(\mathrm{MPa})$ & $\nu_{x}$ & \multicolumn{1}{c}{$\nu_{y}$} \\
\hline Constraining layer (1095 spring steel) & 200,000 & 200,000 & 0.28 & 0.28 \\
Fluidic layer - pure elastomer & 1.2 & 1.2 & 0.48 & 0.48 \\
Fluidic layer - reinforced elastomer & 1.5 & 170 & 0 & 0 \\
Prestressed $90^{\circ}$ EMC & Nonlinear & 170 & 0 & 0 \\
\hline
\end{tabular}

\subsection{Configuration study}

Figure 6 shows the magnitude of curvature $\left(\left|\kappa_{x}^{0}\right|\right)$ of an FPC in each of the possible laminate configurations calculated as a function of actuation pressure for EMC prestrain ranging from 0.3 to 0.6. In all cases, a higher EMC prestrain yields a higher composite curvature about the $Y$ axis for a given actuation pressure. In the unactuated state, curvature is the least in case 1 and and is maximum in case 2 for a given EMC prestrain. A direct coupling between a constraining layer and an EMC enables the EMC's strain energy to be manifest primarily as a deflection $\left(w_{0}\right)$ in the composite in the $Z$ direction; the in-plane component $\left(\partial u / \partial x\right.$ in (1)) of $\epsilon_{x}$ in the constraining layer is negligible. Curvature is lowest in case 1 since a part of the EMC's strain energy is lost to in-plane compression of the fluidic layer. Curvature in case 3 is higher than in case 1 since the mechanics of the constraining layer are minimally affected by the fluidic layer bonded to it. In case 2 , composite deflection is augmented by the in-plane compressive strain $(\partial u / \partial x)$ in the sandwiched fluidic layer, resulting in the highest curvature among the three cases.

Pressurization of the fluid channel results in a reduction in composite curvature in cases 1 and 2. Recognizing that the curvature corresponding to a flat shape is zero, it is apparent that the actuation pressure required to nearly flatten the composite is lower in case 1 . The difference in response is attributed to the larger in-plane compressive strain in the fluidic layer in case 2 . In case 3 , actuation of the composite leads to an increase in its curvature. Deflection of the composite in the $Z$ direction is accompanied by an in-plane tensile strain in the fluidic layer.

For minimal actuation effort at a given EMC prestrain, an FPC must have a higher curvature in the unactuated state with a laminate arrangement as in case 1 and must be actuated to produce a lower curvature. At lower values of EMC prestrain, the actuation pressure required to flatten the composite is lower. However, the trade-off in lowering the EMC prestrain is a loss in 


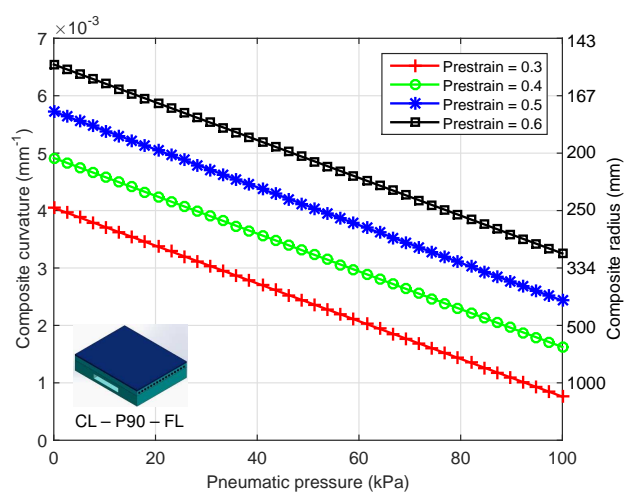

(a)

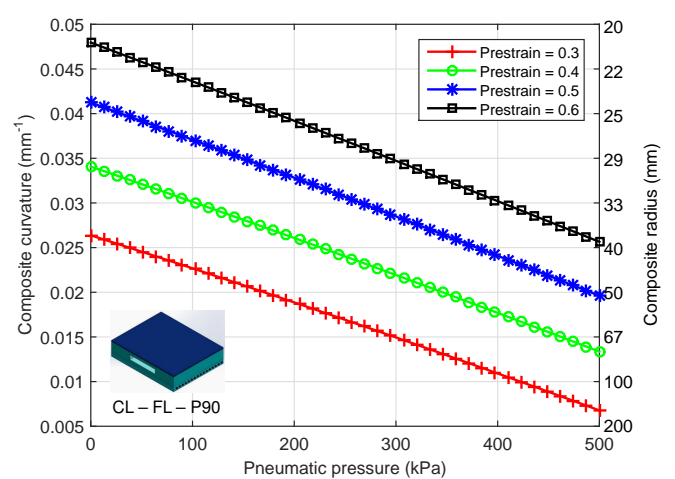

(b)

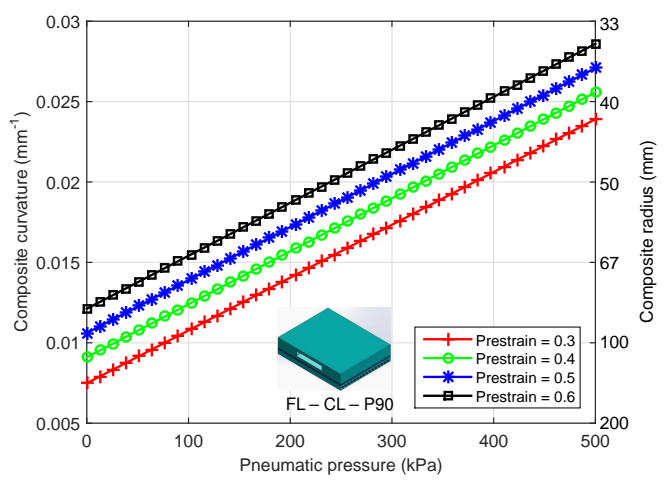

(c)

Figure 6: Modeled curvature vs. actuation pressure of a fluidic prestressed composite in (a) configuration 1, (b) configuration 2, and (c) configuration 3.

morphing ability.

\subsection{Parametric study}

The parameters influencing actuation effort such as thickness, width, and location of the fluid channel are examined in this section. An FPC with laminae arranged as shown in case 1 (Figure 2(b)) is used in this study. Prestrain in the $90^{\circ} \mathrm{EMC}$ is maintained constant at 0.5 .

The relevant design parameters are defined as:

$$
\phi=\frac{C_{y}}{L_{y}}, \quad \chi=\frac{h_{3 a}+h_{3 b}}{h_{2}+h_{3}}, \quad \text { and } \quad \psi=\frac{h_{2}-h_{3 a}}{h_{3 a}+h_{3 b}},
$$

where $\phi, \chi$, and $\psi$ are the non-dimensional width, thickness, and proximity (to the fluidic layer-EMC interface) of the fluid channel. 


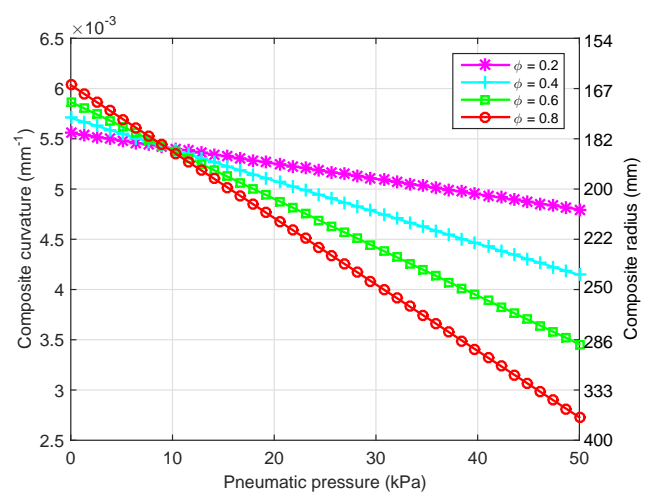

(a)

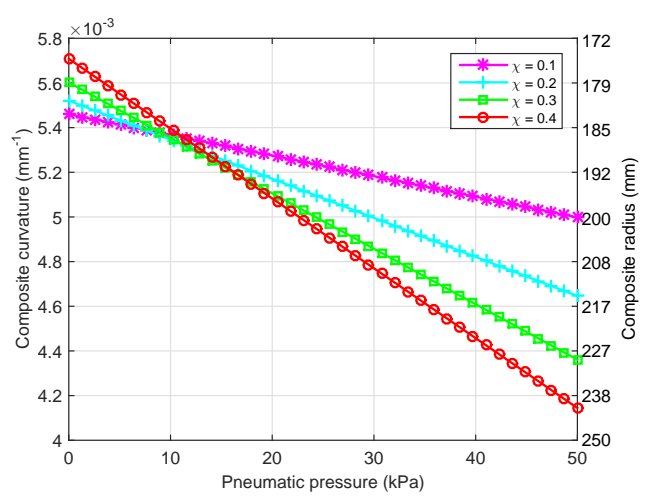

(b)

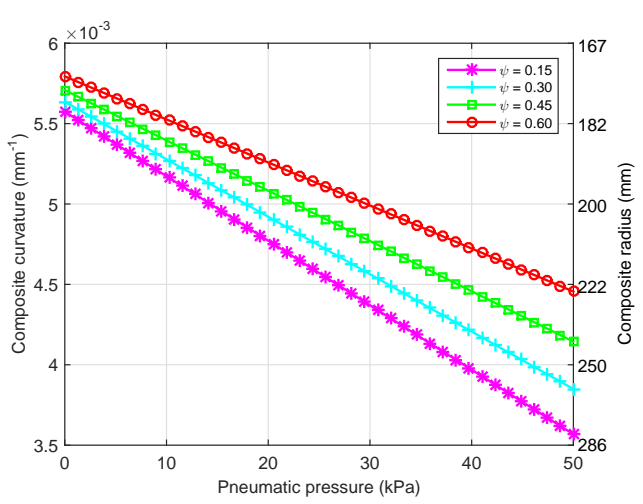

(c)

Figure 7: Response of a fluidic prestressed composite on the non-dimensional (a) width $(\phi)$ for $\chi=0.6$ and $\psi=0.4$, (b) thickness $(\chi)$ for $\phi=0.4$ and $\psi=0.4$, and (c) proximity to the interface $(\psi)$ of the fluid channel for $\phi=0.4$ and $\chi=0.6$.

The response of an FPC for various values of $\phi$ is plotted in Figure 7(a). The values of $\chi$ and $\psi$ are maintained at 0.6 and 0.4 respectively. Based on simulations conducted in an actuation pressure range of $0-50 \mathrm{kPa}$, it is observed that the magnitude of the slope of the response curve increases with an increase in $\phi$. This phenomenon is a consequence of the increase in cross-sectional area of the fluid channel and hence the available force along the channel for a given actuation pressure. The increase in curvature in the unactuated state at higher $\phi$ is due to a reduction of material in the fluidic layer.

The effect of the non-dimensional fluid channel thickness $(\chi)$ on composite response is studied at a constant value of $\phi$ and $\psi$ of 0.4 (Figure 7(b)). An increase in $\chi$ enables a reduction in the actuation pressure needed to obtain 
a given curvature, while increasing the curvature in the unactuated state. Such behavior is explained in the same manner as done in the study on $\phi$. The overall effect of increasing $\phi$ or $\chi$ is an enhanced morphing envelope at a lower actuation pressure.

The dimensions of the fluid channel cross-section are maintained constant $(\phi=0.4, \chi=0.6)$ and the effect of its proximity to the prestressed EMC $(\psi)$ is simulated (Figure $7(\mathrm{c})$ ). The physical equivalent of lowering $\psi$ is a shift in the fluidic layer material towards the bottom face. The effect of this material shift is an increase in compressive stress in the fluidic layer and hence a decrease in composite curvature at a given pressure. Therefore, a decrease in the value of $\psi$ leads to an increase in the slope (magnitude) of the response curve.

Theoretically, the maximum morphing ability in an FPC for a given actuation pressure range is obtained for $\phi \rightarrow 1, \chi \rightarrow 1$, and $\psi \rightarrow 0$. To restrict the strain of the fluid channel in $Y$ and $Z$ directions, the suggested practical limits for $\phi, \chi$, and $\psi$ are $0.6,0.5$, and 0 respectively.

The response of an FPC in various laminate configurations and its dependence on some of the design parameters has been examined using the analytical model presented in section 3. A fabrication procedure for a fluidic prestressed composite is prescribed in the following section.

\section{Composite Fabrication}

Composite beam samples of an FPC in configuration 1 are fabricated to demonstrate its operation and to validate the analytical model. The chosen configuration requires the least actuation effort among the three configurations. Dimensions and material properties of the laminae are the same as listed in Figure 5 and Table 2. The fabrication procedure used for each of the composite laminae is discussed in the following subsections.

\section{1. $90^{\circ} E M C$}

A $90^{\circ}$ EMC is fabricated by sandwiching two layers of unidirectional carbon fibers between two pre-cured silicone rubber sheets. Freshly mixed liquid silicone rubber, applied to the fibers and silicone rubber skins, cures to form an EMC with design specifications listed in Table 3. Rhodorsil 340/CA 45 mold making silicone rubber of durometer grade 45 (shore A) is used as the elastomeric matrix. The same rubber composition is used in the fabrication of all elastomeric elements in the FPC. Unidirectional carbon 
fibers are prepared by removing transverse fibers from woven carbon fabric (Fiberglast Developments Corp., $3.1 \mathrm{~kg} / \mathrm{m}^{2}$ ). A tensile test was conducted on the $90^{\circ}$ EMC up to $110 \%$ strain transverse to the fiber direction with the response shown in Figure 4.

Table 3: Design details of the fabricated $90^{\circ}$ EMC.

\begin{tabular}{crrr}
\hline Material & Density $\left(\mathrm{kg} / \mathrm{m}^{3}\right)$ & Volume fraction & Thickness $(\mathrm{mm})$ \\
\hline Silicone sheet & 1340 & 0.83 & $0.76(\times 2)$ \\
Carbon fibers & 53.53 & 0.17 & 0.50 \\
Total & 1113 & 1 & 2 \\
\hline
\end{tabular}

\subsection{Fluidic layer}

The first step in the fabrication is to build molds for the fluidic layer and the fluid channel. The mold for the fluidic layer is 3D-printed whereas a steel strip is used as a mold for the fluid channel. A release agent (Pattern Release 202, National Engineering Products Inc.) is used on both molds to facilitate the removal of the cured sample. A pre-cured silicone rubber skin is sized to fit the mold for the fluidic layer and then laid out on it. The steel strip is positioned on this rubber skin (Figure 8(a)) and freshly mixed degassed silicone rubber is poured into the mold to create one part of the fluidic layer (Figure 8(b)). Upon curing, the steel strip is removed from the elastomer and the resulting void is filled with paraffin wax. Marchese et al. [35] proposed lost wax casting as a fabrication technique for soft fluidic actuators. A steel tube of diameter $1.78 \mathrm{~mm}(0.07$ ") is used as a pressure supply line and is positioned in the mold as shown in Figure 8(c). The steel tube is locked in position in the composite by smearing its outer surface with a flexible silicone adhesive that cures along with the silicone rubber surrounding it. The outlet in the elastomer is then sealed by pouring silicone rubber around the steel tube (Figure $8(\mathrm{~d})$ ). Curing of the elastomer completes the fabrication of the fluidic layer (Figure 8(e)). Finally, the bottom surface of the fluidic layer in the $X Y$ plane is reinforced with carbon fibers in the $Y$ direction. Fibers wetted with liquid silicone are sandwiched between the bottom face and a pre-cured silicone rubber skin (Figure 8(f)). 


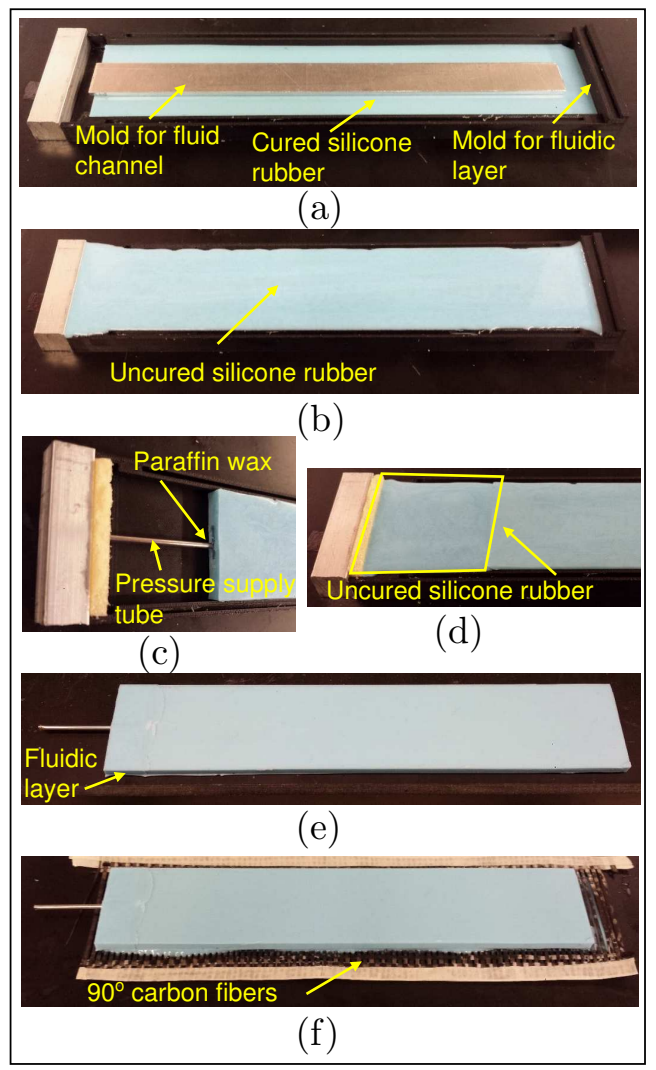

Figure 8: (a) Setup to mold the body of the fluidic layer, (b) layup of silicone rubber to create the body, (c) setup for the end cap, (d) silicone rubber poured to create the end cap, (e) fully cured fluidic layer, (f) fiber-reinforcement of the bottom face.

\subsection{Laminated composite}

The fabricated $90^{\circ}$ EMC layer is held at its ends by grips and prestrained to the desired value. Separately, the fluidic layer and a 1095 spring steel strip that is used as the constraining layer are bonded on either face of the prestressed EMC using a flexible silicone adhesive (DAP Auto-Marine 100\% RTV silicone sealant) and allowed to cure at room temperature for 24 hours (Figure 9(a)). Upon removal from the grips, a fluidic prestressed composite is obtained. At this stage, the composite has a curved shape but the curvature is less than expected due to the restriction offered by the wax (high modulus) in the fluid channel. Finally, the wax (melting point of $57^{\circ} \mathrm{C}$ ) is melted out through the pressure supply line by 


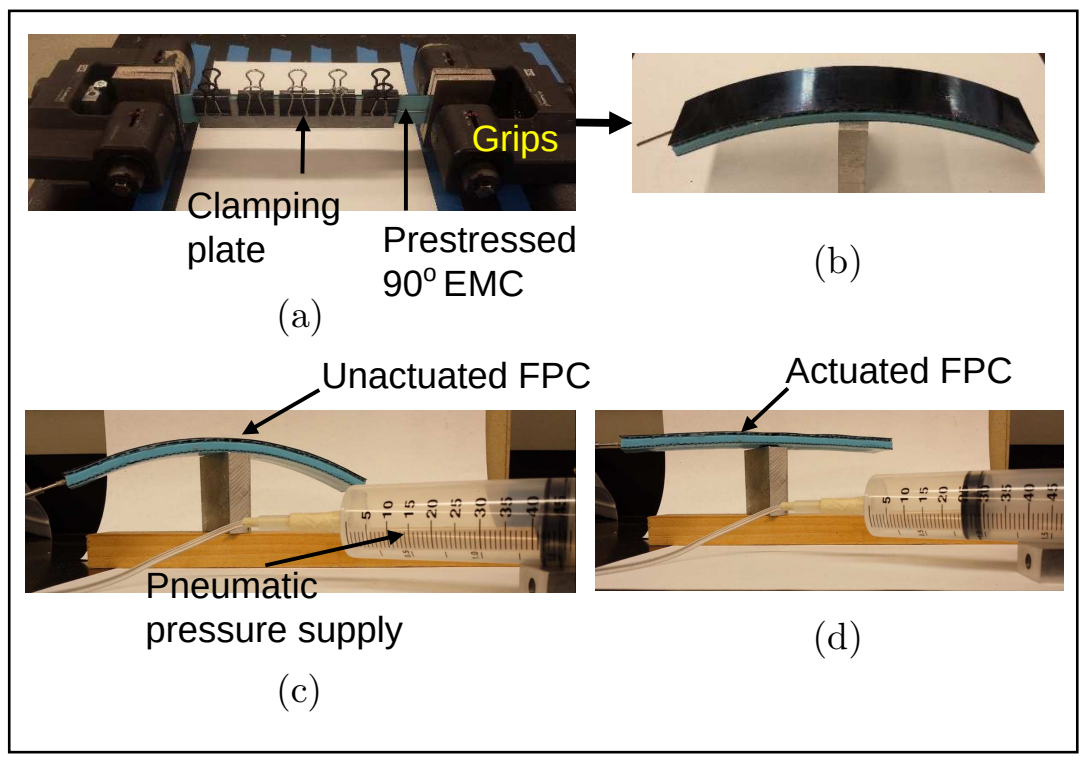

Figure 9: (a) Setup for the lamination of a fluidic prestressed composite, (b) trimmed laminated composite, (c) unactuated and (d) limiting actuated shapes of the composite.

placing the composite in an oven at $60^{\circ} \mathrm{C}$. The resulting composite has a curved shape at equilibrium and can be pressurized to obtain a change in curvature (Figure 9(b)). The composite is actuated pneumatically using a disposable medical syringe to achieve a morphing function. The unactuated and actuated shapes of the fabricated FPC beam are shown in Figures 9(c) and $9(\mathrm{~d})$, respectively. It has been observed that the prestressed $90^{\circ} \mathrm{EMC}$ can peel off from the spring steel strip over an extended period of time. This is probably caused by a high shear stress at the interface created due to a large difference in the elastic moduli of these two layers. In such a case, the roughness of the bonding surfaces influences bond strength. To improve the durability of the bond, a thin layer of paint primer is sprayed onto the metal surface.

\section{Model Validation}

The shape of an FPC beam fabricated in configuration 1 is measured as a function of pneumatic pressure in a quasi-static condition. The experimental setup for this measurement is shown in Figure 10(a). The composite is 
pressurized pneumatically using a plastic medical syringe with a diameter of $28 \mathrm{~mm}$ (1.1") and a stroke of $101.6 \mathrm{~mm}$ (4"). The syringe is rigidly mounted on a base and the position of its piston is set using a threaded rod. The applied pneumatic pressure is measured using a pressure gauge of range $0-103.5 \mathrm{kPa}(0-15 \mathrm{psi})$ that is linked to the syringe using a saddle valve. Reflective markers placed on the concave face of the composite (Figure 10(b)) are tracked using an OptiTrack motion capture system consisting of four cameras that have a resolution of 1.3 megapixels. The composite is mounted on a fixture using thin double-sided tape such that shape change can occur without any restriction while its center remains stationary. Movement of the center does not affect measurement accuracy since only the relative position of markers is required. The coordinates of the markers are then fit to a circle using the method of least squares to calculate the curvature of the composite (Figure 10(c)).

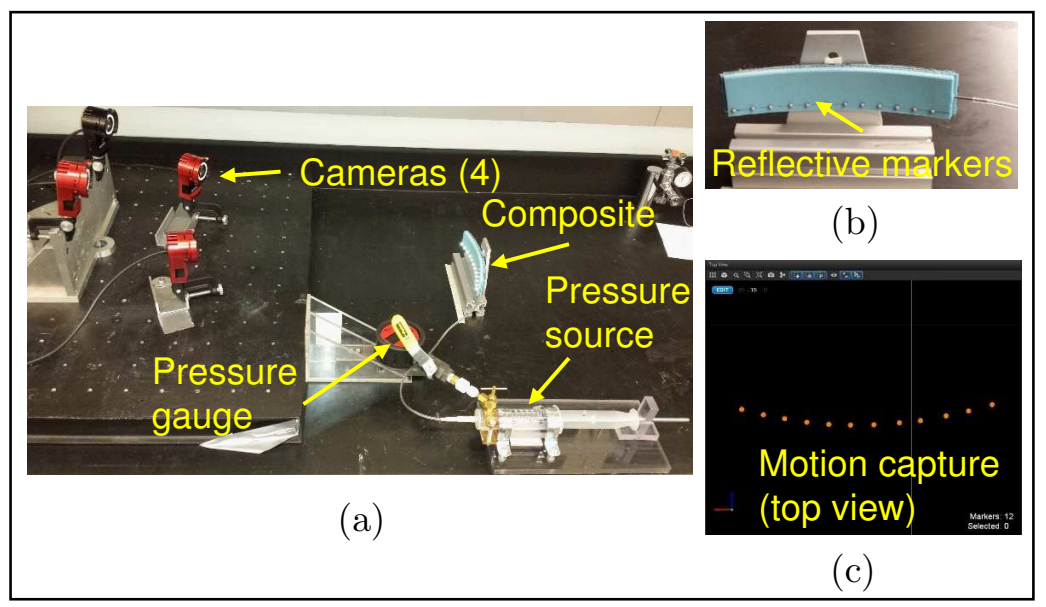

Figure 10: (a) Experimental setup to measure the quasi-static response of a fluidic prestressed composite, (b) composite equipped with reflective markers, (c) spatial coordinates of the reflective markers measured by a motion capture system.

The curvatures of three composite beam samples fabricated with prestrains of $0.25,0.5$, and 0.6 in the $90^{\circ} \mathrm{EMC}$, are measured at discrete values of pneumatic pressure (Figure 11). The maximum applied pressure is restricted to $82.7 \mathrm{kPa}(12 \mathrm{psi})$ due to the limited sealing capability of the plastic syringe, but is sufficient to nearly flatten the composites. It is observed that the measured quasi-static response of an FPC matches fairly well with the 


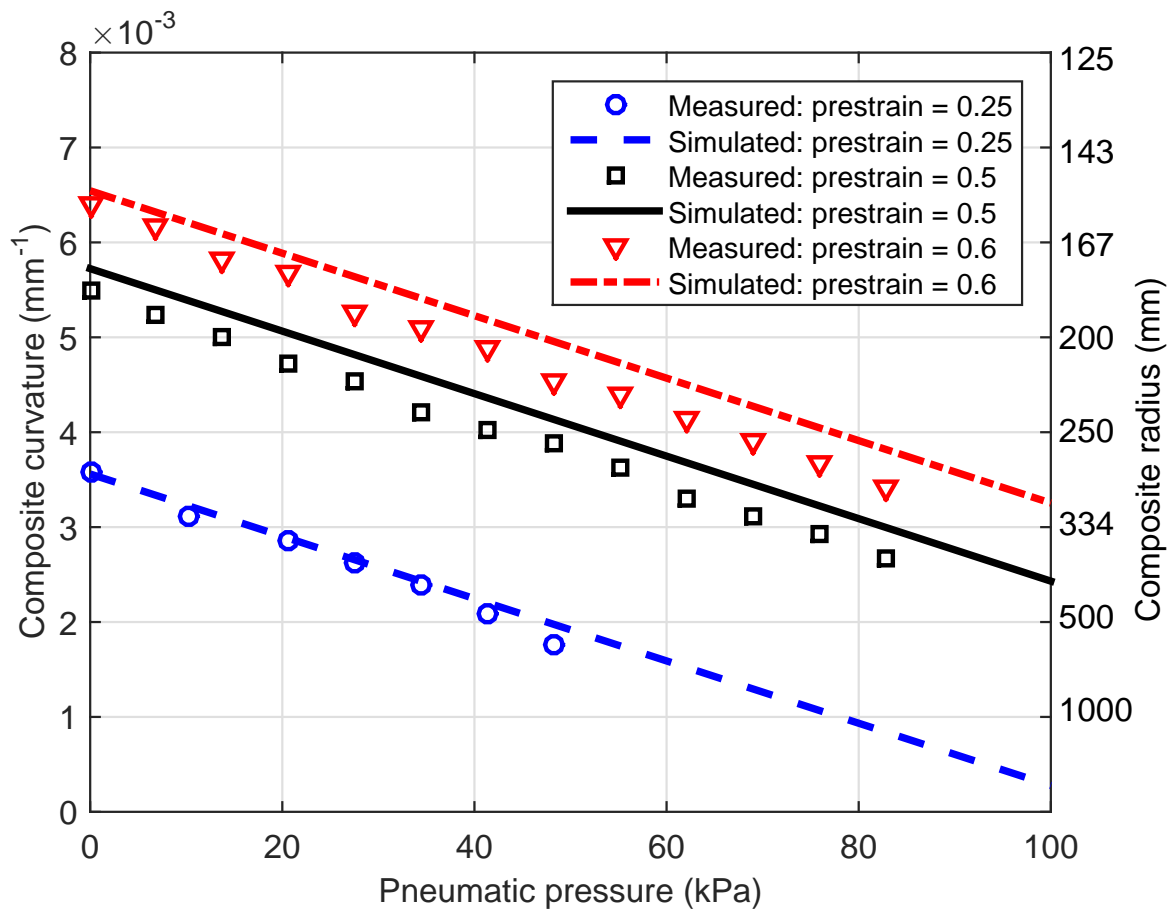

Figure 11: Plot of the curvature of a fluidic prestressed composite (sandwiched $90^{\circ}$ EMC configuration) as a function of actuation pressure for different prestrains in the $90^{\circ} \mathrm{EMC}$.

simulated response. In the unactuated state $(0 \mathrm{kPa})$, the error in curvature is $0.3 \%, 4.0 \%$, and $2.0 \%$ for EMC prestrains of $0.25,0.5$, and 0.6 , respectively. This bias may be attributed to dimensional variability in the fabricated samples. At $82.7 \mathrm{kPa}$, the maximum error in samples with 0.5 and $\mathbf{0 . 6}$ prestrain is $\mathbf{6 . 7 \%}$ and $8.2 \%$ respectively. In the sample with 0.25 prestrain, the maximum error is $10.2 \%$ at $48.2 \mathrm{kPa}$; the composite attains a nearly flat shape at $34.5 \mathrm{kPa}$ where the error is $1.9 \%$. The error in the nearly-flat shape is higher due to the bulging of the bottom face of the fluidic layer. The bulge is a result of the fluid channel cross-section tending towards a circle at higher pressure and is equivalent to a localized increase in composite thickness. As per the parametric study in section 4.2, an increase in fluid channel thickness $(\chi)$ yields an increase in the slope of the response curve. Since the model assumes a plane-stress condition, curvature is over-predicted in the 
simulated response. Bulging may be minimized by lining the inner walls of the fluid channel with an inextensible material such that it expands only in the longitudinal direction.

\section{Concluding Remarks}

A design for a fluidic prestressed composite that has a curved equilibrium geometry in the unactuated state and exhibits a controllable change in shape when actuated, is presented for the first time. An analytical model developed under a plane-stress assumption incorporates the nonlinear behavior of elastomeric layers and simulates the quasi-static response of an FPC accurately at low operating pressures. The laminate configuration of an FPC that requires the least actuation effort is identified and the effects of parameters influencing actuation are examined through a model-based study. A method for fabricating an FPC and measuring its response is presented. The simulated response of the composite is in agreement with the measured response. Durability of FPCs is an area for further investigation and optimization. When used in conjunction with a compact pressure source, fluidic prestressed composites have the potential to serve as lightweight morphing structures that can exhibit drastic, controllable changes in shape.

\section{Acknowledgements}

Financial support was provided by member organizations of the Smart Vehicle Concepts Center, a National Science Foundation Industry/University

Cooperative Research Center (www.SmartVehicleCenter.org). Additional support for S.C. was provided by a Smart Vehicle Center Graduate Fellowship. Technical advice was provided by Dr. Umesh Gandhi and Mr. Kazuhiko Mochida from Toyota Technical Center (TEMA-TTC) in Ann Arbor, MI.

\section{References}

\section{References}

[1] Bowman J, Sanders B, Weisshaar T. Evaluating the impact of morphing technologies on aircraft performance. In: 43rd AIAA/ASME/ASCE/AHS/ASC Structures, Structural Dynamics, and Materials Conference; 2002. p. 1631. 
[2] Barbarino S, Bilgen O, Ajaj RM, Friswell MI, Inman DJ. A review of morphing aircraft. J Intell Mater Syst Struct. 2011;22(9):823-877.

[3] Thill C, Etches J, Bond I, Potter K, Weaver P. Morphing skins. The Aeronautical Journal. 2008;112(1129):117-139.

[4] Daynes S, Weaver PM. Review of shape-morphing automobile structures: concepts and outlook. Proc Inst Mech Eng D: J Automob Eng. 2013;227(11):1603-1622.

[5] Hyer MW. Some Observations on the Cured Shape of Thin Unsymmetric Laminates. J Compos Mater. 1981 Mar;15(2):175-194.

[6] Daynes S, Weaver P. Analysis of unsymmetric CFRP-metal hybrid laminates for use in adaptive structures. Composites Part A. 2010;41(11):1712-1718.

[7] Daynes S, Potter KD, Weaver PM. Bistable prestressed buckled laminates. Compos Sci Technol. 2008;68(15-16):3431-3437.

[8] Daynes S, Diaconu CG, Potter KD, Weaver PM. Bistable prestressed symmetric laminates. J Compos Mater. 2010;44(9):1119-1137.

[9] Lachenal X, Weaver PM, Daynes S. Multi-stable composite twisting structure for morphing applications. Proceedings of the Royal Society A: Mathematical, Physical and Engineering Science. 2012 May;468(2141):1230-1251.

[10] Chillara VSC, Headings LM, Dapino MJ. Self-folding laminated composites for smart origami structures. In: ASME 2015 Conference on Smart Materials, Adaptive Structures and Intelligent Systems; 2015. p. 8968.

[11] Simoneau C, Terriault P, Lacasse S, Brailovski V. Adaptive composite panel with embedded SMA actuators: modeling and validation. Mech Based Des Struc. 2014;42(2):174-192.

[12] Bilgen O, Kochersberger KB, Inman DJ, Ohanian OJ. Novel, bidirectional, variable-camber airfoil via macro-fiber composite actuators. J Aircr. 2010;47(1):303-314. 
[13] Eckstein E, Pirrera A, Weaver PM. Multi-mode morphing using initially curved composite plates. Compos Struct. 2014 Mar;109:240-245.

[14] Lamacchia E, Eckstein E, Pirrera A, Weaver P. Morphing structures: non-linear composite shells with irregular planforms. In: 56th AIAA/ASCE/AHS/ASC Structures, Structural Dynamics, and Materials Conference; 2015. p. 0962.

[15] Huber JE, Fleck NA, Ashby MF. The selection of mechanical actuators based on performance indices. Proc R Soc A. 1997;453(1965):2185-2205.

[16] Chapman EG, Herdic SL, Keller CA, Lynch CS. Development of miniaturized piezo-hydraulic pumps. In: Smart Structures and Materials. International Society for Optics and Photonics; 2005. p. 299-310.

[17] Zhang Z, Philen M. Review: Pressurized Artificial Muscles. J Intell Mater Syst Struct. 2012 Feb;23(3):255-268.

[18] Deimel R, Brock O. A compliant hand based on a novel pneumatic actuator. In: 2013 IEEE International Conference on Robotics and Automation; 2013. p. 2047-2053.

[19] Marchese AD, Onal CD, Rus D. Autonomous soft robotic fish capable of escape maneuvers using fluidic elastomer actuators. Soft Robot. 2014;1(1):75-87.

[20] Philen M, Shan Y, Bakis C, Wang KW, Rahn C. Variable Stiffness Adaptive Structures utilizing Hydraulically Pressurized Flexible Matrix Composites with Valve Control. In: 47th AIAA/ASME/ASCE/AHS/ASC Structures, Structural Dynamics, and Materials Conference; 2006. p. 2134.

[21] Philen M, Shan Y, Wang KW, Bakis C, Rahn C. Fluidic Flexible Matrix Composites for the Tailoring of Variable Stiffness Adaptive Structures. In: 48th AIAA/ASME/ASCE/AHS/ASC Structures, Structural Dynamics, and Materials Conference; 2007. p. 1703.

[22] Feng N, Liu L, Liu Y, Leng J. A bio-inspired, active morphing skin for camber morphing structures. Smart Mater Struct. 2015;24(3):035023. 
[23] Bubert EA, Woods BKS, Lee K, Kothera CS, Wereley NM. Design and fabrication of a passive 1D morphing aircraft skin. J Intell Mater Syst Struct. 2010;21(17):1699-1717.

[24] Murray G, Gandhi F, Bakis C. Flexible matrix composite skins for one-dimensional wing morphing. J Intell Mater Syst Struct. 2010;21(17):1771-1781.

[25] Daynes S, Lachenal X, Weaver PM. Concept for morphing airfoil with zero torsional stiffness. Thin-Walled Struct. 2015;94:129-134.

[26] Shan Y, Philen M, Lotfi A, Li S, Bakis CE, Rahn CD, et al. Variable stiffness structures utilizing fluidic flexible matrix composites. J Intell Mater Syst Struct. 2009;20(4):443-456.

[27] Polygerinos P, Wang Z, Overvelde JTB, Galloway KC, Wood RJ, Bertoldi K, et al. Modeling of soft fiber-reinforced bending actuators. IEEE Trans Robot. 2015;31(3):778-789.

[28] Hyer MW. The room-temperature shapes of four-layer unsymmetric cross-ply laminates. J Compos Mater. 1982;16(4):318-340.

[29] Dano ML, Hyer MW. Thermally-induced deformation behavior of unsymmetric laminates. Int J Solids Struct. 1998;35(17):2101-2120.

[30] Reddy JN. In: Theory and analysis of elastic plates. Philadelphia, PA: Taylor \& Francis; 1999. p. 112.

[31] Peel LD, Jensen DW. Nonlinear modeling of fiber-reinforced elastomers and the response of a "rubber muscle" actuator. Pap-Am Chem Soc: Div Rubber Chem. 2000;(30).

[32] Ogden RW. Large deformation isotropic elasticity - on the correlation of theory and experiment for incompressible rubberlike solids. In: Proc. R. Soc. A. vol. 326; 1972. p. 565-584.

[33] Murugan S, Saavedra Flores EI, Adhikari S, Friswell MI. Optimal design of variable fiber spacing composites for morphing aircraft skins. Compos Struct. 2012;94(5):1626-1633.

[34] Moran MJ, Shapiro HN. In: Fundamentals of engineering thermodynamics. John Wiley \& Sons; 2000. p. 45-46. 
[35] Marchese AD, Katzschmann RK, Rus D. A recipe for soft fluidic elastomer robots. Soft Robot. 2015;2(1):7-25. 